
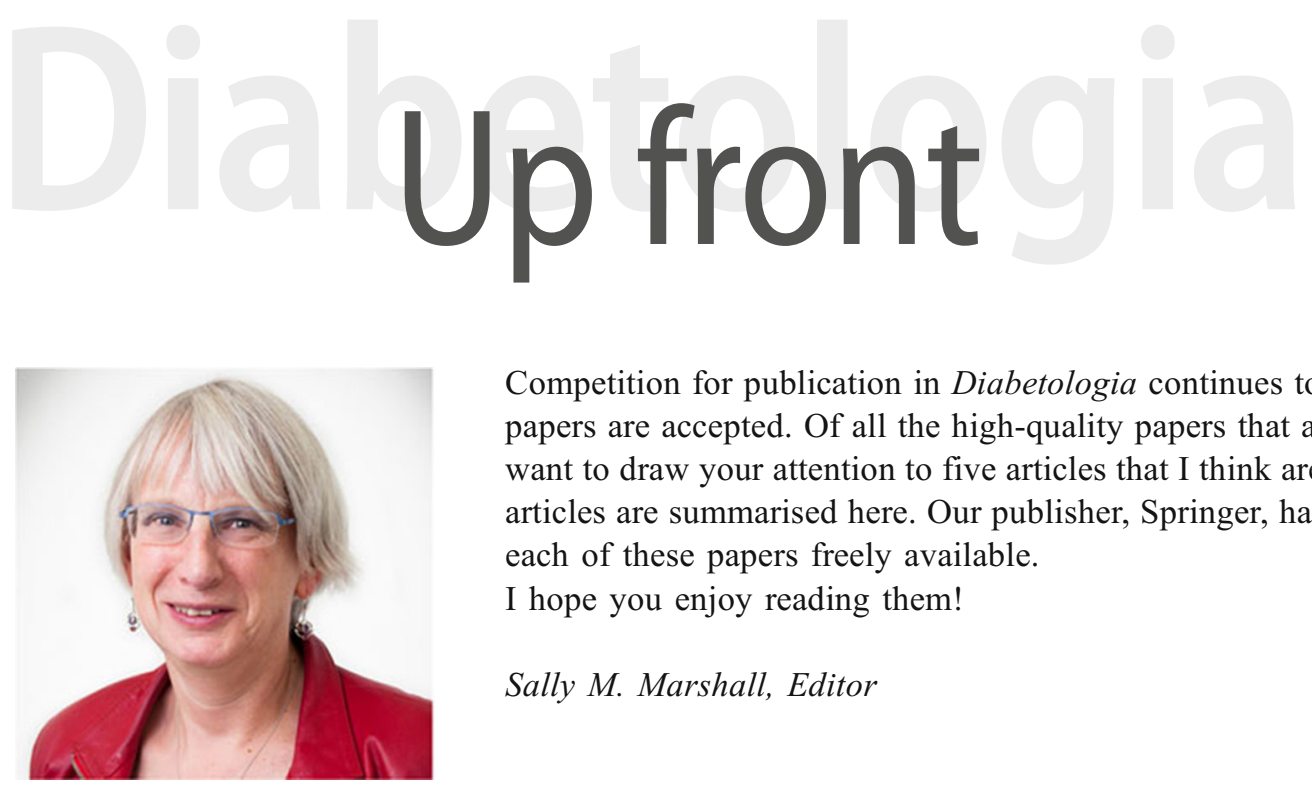

Competition for publication in Diabetologia continues to grow, and less than $20 \%$ of papers are accepted. Of all the high-quality papers that appear in this month's issue I want to draw your attention to five articles that I think are particularly interesting. The articles are summarised here. Our publisher, Springer, has kindly made the full text of each of these papers freely available.

I hope you enjoy reading them!

Sally M. Marshall, Editor

\section{Use of human islets to understand islet biology and diabetes: progress, challenges and suggestions}

Nathaniel J. Hart, Alvin C. Powers

Over the past 15 years, a marked increase in access to human islets for research and work by hundreds of investigators has greatly expanded our understanding of human islet biology. In this issue, Hart and Powers (https://doi.org/10.1007/s00125018-4772-2) highlight and summarise some of these findings, especially those showing similarities and differences between human islets and islets from rodent models of diabetes. In reviewing more than 200 manuscripts reporting research on human islets, published between 2013 and 2017, the authors noted that most publications lacked critical information about the human islets used in experiments, possibly hindering the ability to reproduce and compare experimental outcomes between laboratories. The authors urge the 'human islet research ecosystem' to work cooperatively to develop ways to foster collaboration, transparency and experimental rigour for research using human islets. They suggest a checklist of characteristics and information about human islets that should be reported when these samples are used for research.

(t) The figures from this review are available as a downloadable slideset.

As discussed in an accompanying editorial (https://doi.org/10. 1007/s00125-018-4784-y), Diabetologia and Diabetes have adopted a modified version of Hart and Powers' checklist, to be completed by authors on submission.
The many secret lives of adipocytes: implications for diabetes

Philipp E. Scherer

In the context of the physiological control of systemic metabolism, hardly any other cell type has undergone more of an image change than the adipocyte over the past two decades. Previously viewed mostly as a relatively inert and passive cell with a primary focus on energy storage and release, it is now appreciated for its numerous endocrine functions. In this issue, Philipp E. Scherer (https://doi.org/10.1007/s00125-018-4777$\mathrm{x})$ summarises several of the key aspects that keep the adipocyte at centre stage in the quest for novel endocrine mediators. The author outlines key adipocyte-associated enzymatic targets for the discovery of new therapeutic agents for use in diabetes, specifically those aimed at normalising carbohydrate and lipid metabolism. These factors include adipokines, important lipid-signalling molecules (such as ceramides) and key metabolites (such as uridine). Many of these can be released from adipose tissue in the classical endocrine fashion, or they may be packaged into exosomal vesicles that adipose tissue very effectively releases. The author also comments on the remarkable versatility of the cellular physiology of the adipocyte in terms of its anatomical location, its ability to act as a storage cell (the white adipocyte) or a thermogenic cell (beige and brown adipocytes) and its ability to de-differentiate into adipogenic precursor populations and even myofibroblasts, which are critically involved in fibrotic 
responses. The author concludes that, despite advances in adipocyte knowledge, there are many unresolved issues that await targeted research in order to identify novel means by which disease-associated adipose tissue can be reprogrammed into being the benign protective bystander that it was originally meant to be, before we provided massive insults to the tissue through excess energy intake.

(b) The figures from this review are available as a downloadable slideset.

\section{Afternoon exercise is more efficacious than morning exercise at improving blood glucose levels in individuals with type 2 diabetes: a randomised crossover trial}

Mladen Savikj, Brendan M. Gabriel, Petter S. Alm, Jonathon Smith, Kenneth Caidahl, Marie Björnholm, Tomas Fritz, Anna Krook, Juleen R. Zierath, Harriet Wallberg-Henriksson

Glucose tolerance, insulin sensitivity and skeletal muscle oxidative capacity undergo circadian oscillations, and the interaction of these factors could lead to divergent adaptations to exercise. High-intensity interval training (HIIT) has a beneficial effect on blood glucose concentration in individuals with type 2 diabetes. However, the time of day that results in the strongest adaptations of blood glucose levels to HIIT remains unknown. In this issue, Savikj, Gabriel et al (https://doi.org/10. 1007/s00125-018-4767-z) report on a randomised crossover trial of 11 men with type 2 diabetes. They demonstrate that afternoon HIIT is more efficacious than morning HIIT at lowering blood glucose values in this cohort. Furthermore, morning HIIT had early deleterious effects on blood glucose values. The authors' data highlight the importance of optimising the timing of exercise when prescribing HIIT in the clinical management of type 2 diabetes.

Skin autofluorescence predicts incident type 2 diabetes, cardiovascular disease and mortality in the general population

Robert P. van Waateringe, Bernardina T. Fokkens, Sandra N. Slagter, Melanie M. van der Klauw, Jana V. van VlietOstaptchouk, Reindert Graaff, Andrew D. Paterson, Andries J. Smit, Helen L. Lutgers, Bruce H. R. Wolffenbuttel

Autofluorescence measurements are based on the detection of AGEs in the skin. High skin autofluorescence is associated with high risk of cardiovascular complications and mortality in people with type 2 diabetes, as well as renal failure. However, whether this non-invasive measurement can be used as a predictive biomarker in the general population has not been elucidated. In this issue, van Waateringe et al (https://doi.org/10.1007/s00125-0184769-x) examined whether measurement of skin autofluorescence can predict 4 year risk of incident type 2 diabetes, cardiovascular disease (CVD) and mortality in the general population. They report that a higher skin autofluorescence is associated with a higher risk of developing type 2 diabetes and CVD in people free of these diseases at baseline. In addition, they found that baseline skin autofluorescence was elevated in individuals who had died over the follow-up period, compared with individuals who survived. These findings were independent of other important risk factors, such as blood glucose, $\mathrm{HbA}_{1 \mathrm{c}}$ and classical risk factors, including high blood pressure, high cholesterol and smoking. These findings suggest that measurement of skin autofluorescence is of clinical value for screening for future risk of type 2 diabetes, CVD and mortality and can improve risk estimates for these conditions.

A variant of the glucose transporter gene $S L C 2 A 2$ modifies the glycaemic response to metformin therapy in recently diagnosed type 2 diabetes

Wolfgang Rathmann, Klaus Strassburger, Brenda Bongaerts, Oliver Kuss, Karsten Müssig, Volker Burkart, Julia Szendroedi, Jörg Kotzka, Birgit Knebel, Hadi Al-Hasani, Michael Roden, for the GDS Group

Genetic factors play a role in the highly variable glycaemic response to metformin. The $S L C 2 A 2$ gene encodes the glucose transporter isoform GLUT2. In this issue, Rathmann et al (https://doi.org/10.1007/s00125-018-4759-z) investigated the interaction between the single nucleotide polymorphism rs8192675 in SLC2A2 and glycaemic response to metformin (reduction of fasting glucose) in individuals with recently diagnosed type 2 diabetes. Among a cohort of 508 adults with type 2 diabetes, $\mathrm{C}$ allele carriers (TC/CC genotypes) more frequently presented with diabetes symptoms (polyuria, thirst) at diagnosis. During the first year after diagnosis, $\mathrm{C}$ allele carriers on metformin monotherapy showed a greater reduction in fasting glucose compared with individuals homozygous for the $\mathrm{T}$ allele, after adjusting for age, sex, BMI, diabetes duration and baseline glucose. In addition, in the metformin therapy group, $\mathrm{C}$-peptide secretion was higher in individuals with TC/CC genotypes than in individuals with the TT genotype. These findings indicate that a variant in the $S L C 2 A 2$ gene (encoding GLUT2) modifies the glycaemic response to metformin in individuals recently diagnosed with type 2 diabetes.

Publisher's Note Springer Nature remains neutral with regard to jurisdictional claims in published maps and institutional affiliations.

All text supplied by the authors. 